\title{
NILPOTENT SUBGROUPS OF CLASS 2 IN FINITE GROUPS
}

\author{
LUCA SABATINI
}

In memory of Carlo Casolo (1958-2020)

\begin{abstract}
We show that every finite group $G$ of size at least 3 has a nilpotent subgroup of class at most 2 and size at least $|G|^{1 / 32} \log \log |G|$. This answers a question of Pyber, and is essentially best possible.
\end{abstract}

\section{INTRODUCTION}

While dealing with a finite group, it is a question of great interest whether it has some large abelian/nilpotent/solvable subgroup. In 1976, Erdös and Straus 2 proved that every finite group $G$ has an abelian subgroup of size roughly log $|G|$. In [7, Pyber showed that this can be improved to $2^{\varepsilon \sqrt{\log |G|}}$, for some fixed $\varepsilon>0$. In the same article, a key proposition shows that every finite group $G$ has a solvable subgroup of size at least $|G|^{\varepsilon / \log \log |G|}$ for some fixed $\varepsilon>0$, and the inequality is sharp. In this paper, we prove that actually every finite group has a such large nilpotent subgroup of class at most 2 .

Theorem 1. Every finite group $G$ of size at least 3 has a nilpotent subgroup of class at most 2 and size at least $|G|^{1 / 32 \log \log |G|}$.

By a classical result of Dixon 11, a solvable subgroup of the symmetric group on $n$ elements has size at most $24^{(n-1) / 3}$. This shows that the "log log" factor cannot be replaced by anything smaller.

All the logarithms in this paper are to base 2.

\section{Proof of Theorem 1}

The key tool to find nilpotent subgroups of class at most 2 is the following generalization of a remark of Thompson 8 . We remind the reader that a finite group lies in this class if and only if its derived subgroup is contained in its center.

Lemma 2. Let $G$ be a finite group, and let $H \leqslant G$ be of minimal size among the subgroups of $G$ which provide an abelian section of maximal order. Then $H$ is nilpotent of class at most 2.

Proof. We first show that $H$ is nilpotent. If not, take $M \leqslant H$ a non-normal maximal subgroup of $H$. Then $M H^{\prime}=H$ or $M H^{\prime}=M$, but the second is impossible because $M$ is not normal in $H$. It follows that

$$
\left|H / H^{\prime}\right|=\left|M H^{\prime} / H^{\prime}\right|=\left|M / M \cap H^{\prime}\right| \leq\left|M / M^{\prime}\right| \text {. }
$$

' Since $|M|<|H|$, then one can conclude that $H$ is nilpotent, i.e. it is the direct product of its Sylow's subgroups $P_{1}, \ldots, P_{k}$. We show that each of these has nilpotency class at most 2. Since both the derived subgroup and the center factorize through a direct product, the proof shall follow. Now $H$ has the property that $H / H^{\prime}$ is larger than every other abelian section of $H$. We see that $P_{i}$ has the same property, for every $1 \leq i \leq k$. In fact, if not, then since $H / H^{\prime} \cong P_{1} /\left(P_{1}\right)^{\prime} \times \ldots \times P_{k} /\left(P_{k}\right)^{\prime}$, an abelian section of $H$ of size larger than $H / H^{\prime}$ would be found. Then, the proof follows from [8, Lemma].

A finite nilpotent group $G$ of class at most 2 has an abelian section of size at least $|G|^{1 / 2}$. Thus, a large nilpotent subgroup of class at most 2 and a large abelian section are essentially equivalent. At the end of [7 Pyber remarks that every finite group $G$ has an abelian section of size at least $|G|^{\varepsilon /(\log \log |G|)^{2}}$ for some fixed $\varepsilon>0$, and wonders whether much more is true. Of course, Theorem 1 fullfils this purpose by showing that one "log log" factor can be dispensed with.

We say that a finite group is of alternating type if all its nonabelian composition factors are alternating of degree at least 23 (alternating factors of smaller degree are excluded for technical reasons). We report the following useful result.

Lemma 3 (Corollary 2.3 (b) in [7). Every finite group $G$ contains an alternating type subgroup of size at least $|G|^{1 / 5}$.

Pyber proves Lemma 3 with 8 in place of 23, but his argument can be easily extended up to this threshold. Let us spend a few words explaining how this is obtained. Looking in a chief series $1=G_{0} \triangleleft G_{1} \triangleleft \ldots \triangleleft G_{r}=G$, we remove the factors $G_{i} / G_{i-1}$ which are direct products of large alternating groups. It is always possible, inside each of the remaining factors, to find a very large intravariant solvable subgroup (remark that Alt(22), but not Alt(23), contains a 3-subgroup

2020 Mathematics Subject Classification. primary 20D15, 20 F69. 
which is sufficiently large). Then one can merge these solvable sections and the full alternating factors, using a quite technical result of Chunikhin.

Let $\left(a_{i}\right)_{i \geq 1}$ be a sequence of distinct integers greater than 6 , and let $\left(b_{i}\right)_{i \geq 1}$ be a sequence of positive integers. Let $H:=\prod_{i} \operatorname{Alt}\left(a_{i}\right)^{b_{i}}$. The next is a simple remark.

Lemma 4. $\operatorname{Aut}(H) \cong \prod_{i} \operatorname{Sym}\left(a_{i}\right) \imath_{b_{i}} \operatorname{Sym}\left(b_{i}\right)$.

Proof. For every $i$, we have that $\operatorname{Alt}\left(a_{i}\right)^{b_{i}}$ is a characteristic subgroup of $H$. It follows that $\operatorname{Aut}(H) \cong \prod_{i} \operatorname{Aut}\left(\operatorname{Alt}\left(a_{i}\right)^{b_{i}}\right)$. Since $a_{i} \geq 7$, it is easy to see that $\operatorname{Aut}\left(\operatorname{Alt}\left(a_{i}\right)^{b_{i}}\right) \cong \operatorname{Sym}\left(a_{i}\right) \imath_{b_{i}} \operatorname{Sym}\left(b_{i}\right)$ for every $i$, as desired.

We now prove Theorem 1 in the range of solvable groups.

Lemma 5. Every finite solvable group $G$ of size at least 3 contains an abelian section of size at least $|G|^{1 / 4 \log \log |G|}$. Therefore it contains a such large nilpotent subgroup of class at most 2.

Proof. From [4. Corollary] we have that $G$ contains a nilpotent subgroup $H$ of size at least $|G|^{1 / 3}$. The classical result [3. Theorem 2.54] says that a finite $p$-group of nilpotency class $c \geq 1$ has derived length at most $1+\log c$, and this is easily extended to every finite nilpotent group. So if $c$ is the nilpotency class of $H$, then $H$ has derived length at most $1+\log c \leq 1+\log \log |H|$. Using pigeonhole on the derived series, we find an abelian section of size at least

$$
|H|^{1 /(1+\log \log |H|)} \geq|G|^{1 /\left(3+3 \log \left(3^{-1} \log |G|\right)\right)} \geq|G|^{1 / 4 \log \log |G|},
$$

where we used that $x^{1 /(1+\log \log x)}$ is an increasing function when $x \geq 3$.

The second part follows from Lemma 2

It is worth noticing that, unlike Theorem 1 perhaps Lemma 5 could be improved significantly. The next conjecture is really equivalent to [5, Problem 14.76].

Conjecture 6. There exists an absolute constant $\varepsilon>0$ such that every finite solvable group $G$ has a nilpotent subgroup of class at most 2 and size at least $|G|^{\varepsilon}$.

Proof of Theorem 1. Up to a factor of $1 / 5$, we can use Lemma 3 to replace an arbitrary finite group with a group of alternating type. Moreover, for groups of cardinality less than 20, the desired subgroup can be found trivially. So let $G$ be a group of alternating type and size at least 20. Let us denote by $\operatorname{Sol}(G)$ the solvable radical of $G$, i.e. the largest solvable normal subgroup. First suppose that $|\operatorname{Sol}(G)|>|G|^{12 / 19}$. Thus, from Lemma 5 , $\operatorname{Sol}(G)$ and then $G$ has an abelian section of size at least

$$
|\operatorname{Sol}(G)|^{1 / 4 \log \log |\operatorname{Sol}(G)|} \geq|G|^{3 / 19 \log \log |G|}
$$

and so we are done. Otherwise, if $|\operatorname{Sol}(G)| \leq|G|^{12 / 19}$, then $\widetilde{G}:=G / \operatorname{Sol}(G)$ has size at least $|G|^{7 / 19}$. Moreover, $\widetilde{G}$ has no non-trivial solvable normal subgroups. Let $H:=\operatorname{Soc}(\widetilde{G})$ be the subgroup generated by the minimal normal sugroups. Since $\widetilde{G}$ has alternating type, we have $H \cong \prod_{i} \operatorname{Alt}\left(a_{i}\right)^{b_{i}}$ for some $a_{i} \geq 23$ and $b_{i} \geq 1$. It is well known that $C_{\widetilde{G}}(H) \leqslant H$,

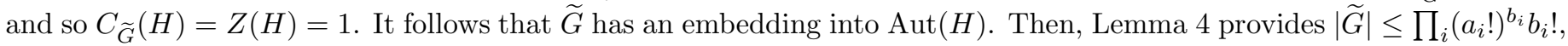
and $\log |\widetilde{G}| \leq \sum_{i} b_{i}\left(a_{i} \log a_{i}+\log b_{i}\right)$. On the other hand $\widetilde{G}$ contains a copy of $\prod_{i} \operatorname{Alt}\left(a_{i}\right)^{b_{i}}$. For every $i$, $\operatorname{Alt}\left(a_{i}\right)$ contains a 3-elementary abelian subgroup of size $3^{\left\lfloor a_{i} / 3\right\rfloor} \geq 2^{3 a_{i} / 7}$. This shows that $H$ and then $\widetilde{G}$ contains an abelian subgroup of size at least $\prod_{i} 2^{3 a_{i} b_{i} / 7}$. The logarithm of this quantity is $\frac{3}{7} \sum_{i} a_{i} b_{i}$. For every $i$ we have

$$
a_{i} b_{i} \geq \frac{b_{i}\left(a_{i} \log a_{i}+\log b_{i}\right)}{\log b_{i}+\log a_{i}} \geq \frac{b_{i}\left(a_{i} \log a_{i}+\log b_{i}\right)}{\log \left(b_{i}\left(a_{i} \log a_{i}+\log b_{i}\right)\right)} .
$$

It follows that

$$
\begin{aligned}
\sum_{i} a_{i} b_{i} & \geq \frac{\sum_{i} b_{i}\left(a_{i} \log a_{i}+\log b_{i}\right)}{\max _{i}\left(\log \left(b_{i}\left(a_{i} \log a_{i}+\log b_{i}\right)\right)\right)} \\
& \geq \frac{\sum_{i} b_{i}\left(a_{i} \log a_{i}+\log b_{i}\right)}{\log \left(\sum_{i}\left(b_{i}\left(a_{i} \log a_{i}+\log b_{i}\right)\right)\right)} \\
& \geq \frac{\log |\widetilde{G}|}{\log \log |\widetilde{G}|} .
\end{aligned}
$$

To sum up, we proved that $\widetilde{G}$ and then $G$ contains an abelian section of size at least

$$
|\widetilde{G}|^{3 / 7 \log \log |\widetilde{G}|} \geq|G|^{3 / 19 \log \log |G|},
$$

and the proof is complete via Lemma 2 once we put back the factor $1 / 5$ in (2.1) and (2.2). 
Remark 7. We would like to make a remark about the gap between Pyber's $2^{\varepsilon \sqrt{\log |G|}}$ bound and the much larger $|G|^{1 / 32 \log \log |G|}$. This difference is explained by the "distance" between nilpotent groups of class 2 and abelian groups (in contrast to the "proximity" between solvable groups and nilpotent groups of class 2, for example). By a non-explicit construction of Ol'shanskii [6], there exists an arbitrarily large finite group $G$, nilpotent of class 2, without abelian subgroups larger than $2^{\alpha \sqrt{\log |G|}}$ for some fixed $\alpha>0$.

\section{REFERENCES}

1. J.D. Dixon, The Fitting subgroup of a linear solvable group, Journal of the Australian Mathematical Society 7 (1967), $417-424$.

2. P. Erdős, E.G. Straus, How abelian is a finite group?, Linear and Multilinear Algebra 3 (1976), 307-312.

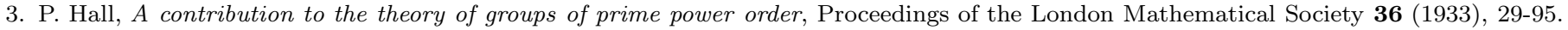

4. H. Heineken, Nilpotent subgroups of finite solvable groups, Archiv der Mathematik 56 (1991), $417-423$.

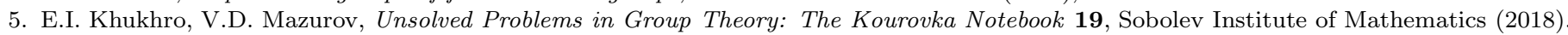

6. A.Y. Ol'shanskii, The number of generators and orders of abelian subgroups of finite p-groups, Mathematical Notes 23 (1978), $183-185$.

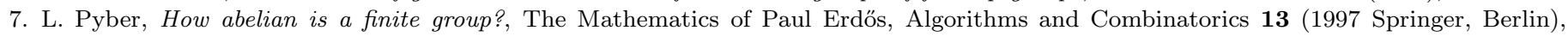
$372-384$

8. J. Thompson, A replacement theorem for p-groups and a conjecture, Journal of Algebra 13 (1969), $149-151$.

Luca Sabatini, Dipartimento di Matematica e Informatica "Ulisse Dini", University of Firenze, Viale Morgagni 67/A, 50134 Firenze, Italy

Email address: luca.sabatini@unifi.it 